\title{
STATISTICAL MECHANICS OF A LIPID MONOLAYER
}

\author{
A J. KOX \\ Institute of Theoretical Phystcs, Untversity of Amsterdam, Amsterdam, The Netherlands \\ and \\ F.W WIEGEL \\ Department of Applied Physics, Twente University of Technology, \\ Enschede, The Netherlands
}

Received 10 January 1978

\begin{abstract}
We calculate from first principles the equation of state of a simple type of membrane a monolayer consistıng of lipid chain molecules with short-range repulsive and long-range attractive forces An approximate solution to the packing problem of the hydrocarbon chains is obtained by using a mathematical analogy with the quantum gas of hard disks. The long-range attractive forces are incorporated in a mean-field approximation The results are in qualitative agreement with experiments.
\end{abstract}

\section{Introduction}

The fact that the structural framework of biological membranes is formed by a bilayer of lipid molecules is by now fairly well established. For that reason lipid bilayers, and also lipid monolayers, have been widely studied ${ }^{1,2}$ ). Experiments on both lipid and biological membranes (which apart from lipids also incorporate large globular proteins) have shown that these systems undergo several phase transitions ${ }^{3.4}$ ). Qualitatively the same behaviour is found in monolayers of lipids. Since, moreover, theoretical considerations suggest that the properties of monolayers and bilayers are related in a transparent way ${ }^{5,6}$ ), theoretical study of the former may lead to a better understanding of the structure and function of biological membranes.

Most authors agree on the fact that two or three different phase transitions occur in both monolayers and bilayers. The mechanism of these transitions may be understood by considering the molecular structure of these monolayers. A lipid molecule consists of a polar, hydrophilic head group, and one or two hydrophobic hydrocarbon chains. In the high density case the chains of the molecules will be fully extended, more or less parallel to each other and tilted with respect to the surface of the fluid (usually water) on 
which the layer rests. All carbon-carbon bonds are in the low energy trans conformation; the heads are arranged in a regular (probably hexagonal) array on the surface of the fluid. Lowering the density increases the mobility of the tails and thus the possibility of the bonds to assume one of the two gauche conformations, which have a higher energy. This effect is observed as a phase transition (the "chain melting" transition). In most systems this transition is preceded by a smaller one which is of unclear origin. Some authors ${ }^{5,7,8}$ ) believe that disordering of the head groups plays a role in this transition; according to a recent investigation ${ }^{9}$ ) it is associated with the formation of ripples in the layer. The chain melting transition has been the subject of much study, both theoretically and experimentally. We, too, shall be concerned with this transition, which sometimes is said to occur between the "liquid-condensed" and "liquid-expanded" states.

Lowering the density beyond the chain melting transition leads to a phase transition from the liquid-expanded to a "gaseous" state. The density in the latter state is very low, the molecules are far apart, and the tails are lying more or less flat on the surface. This transition, too, has been studied experimentally: critical parameters and exponents have been measured with high accuracy ${ }^{10,11}$ ).

A statistical mechanical theory of lipid monolayers should explain the form of the observed isotherms and the nature of the different phases and of the phase transitions in terms of the intermolecular interactions. The development of such a statistical mechanical theory is inhibited by two complicating circumstances.

(a) Two neighbouring $\mathrm{C}-\mathrm{C}$ bonds in a hydrocarbon chain can be oriented in several ways with respect to each other. This fact causes each hydrocarbon chain to have a very large number of configurations. Owing to the short-range repulsion between the different hydrocarbon chains in the monolayer the calculation of the total number of non-overlapping chain configurations leads to a difficult combinatorial problem.

(b) The monolayer is held together by long-range attractive forces which act mainly between the hydrocarbon tails of the molecules.

To our knowledge the first attempt to derive the isotherm of a monolayer of chain molecules is due to Ohki ${ }^{12}$ ). This author neglected the mobility of the hydrocarbon chains and represented the attractive interaction by a nearest neighbour interaction. Thus, in this model the lipids are hard rods with short-range attractions.

In Scott's first paper ${ }^{13}$ ) Ohki's assumption of rigid molecules is slightly relaxed by permitting three states for one chain: one trans and two gauche states. This model shows some rotational disordering at high temperatures, but is nevertheless unrealistic because not just one but each $\mathrm{CH}_{2}$ group in a chain has three available conformations. The treatment of the packing problem (a) is therefore inadequate. In his second paper $^{8}$ ) the number of permitted states per chain is increased to a maximum of 14 ; this leads to a more realistic treatment of the repulsive interactions. 
Marsh $^{14}$ ) tries to solve the packing problem by assuming that every configuration of a hydrocarbon chain which contains two neighbouring gauche conformations is forbidden. Hence this theory, like Scott's, replaces the many-chain excluded volume problem by a one-chain counting problem.

Similar approximations are made by Jacobs et al. ${ }^{15}$ ), McCammon and Deutch $^{16}$ ) and Jackson ${ }^{17}$ ). However, these authors also include repulsive interactions in their models.

In Marcelja's theory ${ }^{5}$ ) all configurations of one chain are generated numerically. The interactions with neighbouring chains are taken into account in an effective field approximation only.

A more phenomenological approach has been followed by Stoeckly ${ }^{18}$ ). This author assumes that, even at low densities, the molecules form tightly bound clumps so that the monolayer may be treated as a gas of clumps.

Finally we should point out that, although the statistical mechanics of monolayers in a space of three dimensions can only be developed approximately, the analogous problem in a two-dimensional space can be solved in full detail, as has been shown by $\mathrm{Nagle}^{7,19,20,6,21}$ ) and Wiegel ${ }^{22-24}$ ). These exact solutions will often provide clues to the behaviour of the threedimensional membrane. A semiphenomenological extension of Nagle's model to three dimensions has recently been given by Priest ${ }^{25}$ ).

The present paper contains what we believe to be the first attempt to deal with problems (a) and (b) in a systematic way. Sections 2 and 3 are devoted to problem (a), and section 4 to problem (b). A critical discussion of our results is given in section 5. A brief summary of this work has appeared elsewhere ${ }^{26}$ ).

\section{The phase space of a hydrocarbon chain}

In this section we shall be concerned with the phase space of a single fatty acid molecule in a monolayer. Every carbon-carbon bond in its chain has three possible conformations with respect to its two immediate predecessors: one trans conformation to which we assign zero energy and two gauche conformations (with equal energy $\epsilon>0$ ). A rough estimate is ${ }^{27}$ )

$$
\epsilon \cong 0.35 \times 10^{-13} \mathrm{erg} .
$$

At physiological temperatures this energy is comparable in order of magnitude to the average rotational kinetic energy of a bond, which equals $\frac{1}{2} k_{\mathrm{B}} T=$ $0.69 \times 10^{-16} \times T$ erg. This implies that at biologically relevant temperatures the $\mathrm{C}-\mathrm{C}$ bonds of the hydrocarbon chains in the membrane are highly moveable, hindered in their rotation by excluded volume constraints only.

Let us choose a system of coordinates in such a way that the plane $z=0$ coincides with the surface of the monolayer. The $(x, y)$ coordinates of the successive $C$ atoms in the chain are given by the sequence

$$
\left(x^{(0)}, y^{(0)}\right),\left(x^{(1)}, y^{(1)}\right), \ldots,\left(x^{(N)}, y^{(N)}\right),
$$


where $\left(x^{(0)}, y^{(0)}\right)$ denotes the coordinates of the head group. This sequence will be interpreted as a discrete random walk in the $(x, y)$ plane, starting out at the position of the head group and reaching the end point after $N$ steps. It is well known ${ }^{28}$ ) from the theory of random walks that for $N \gg 1$ the probability density for the endpoint $P\left(x^{(N)}, y^{(N)}\right)$ is the solution of the diffusion equation

$$
\left[\frac{\partial}{\partial N}-D\left(\frac{\partial^{2}}{\partial x^{(N) 2}}+\frac{\partial^{2}}{\partial y^{(N) 2}}\right)\right] P\left(x^{(N)}, y^{(N)}\right)=0 .
$$

Here $D$ is the diffusion coefficient, the temperature dependence of which follows from the details of the underlying model. It is easy to verify that the solution of (3) is given by

$$
P\left(x^{(N)}, y^{(N)}\right)=(4 \pi N D)^{-1} \exp \left[-\frac{1}{4 N D}\left\{\left(x^{(N)}-x^{(0)}\right)^{2}+\left(y^{(N)}-y^{(0)}\right)^{2}\right\}\right] .
$$

The average squared displacement per step equals $4 D$. The probability $P\left(x^{(N)}, y^{(N)}\right)$ equals the ratio of two configuration integrals:

$$
P\left(x^{(N)}, y^{(N)}\right)=\frac{Q\left(x^{(N)}, y^{(N)}\right)}{\int Q\left(x^{(N)}, y^{(N)}\right) \mathrm{d} x^{(N)} \mathrm{d} y^{(N)}},
$$

where $Q\left(x^{(N)}, y^{(N)}\right)$ denotes the sum over all configurations with fixed initial position $\left(x^{(0)}, y^{(0)}\right)$ and fixed final position $\left(x^{(N)}, y^{(N)}\right)$. The denominator can be calculated as follows. Consider two successive $\mathrm{C}-\mathrm{C}$ pairs and introduce polar coordinates such that the first bond has azimuth $\theta=0$. The interaction energy of the two bonds $V(\phi)$ is a function of the polar angle $\phi$ only, with three minima corresponding to one trans and two gauche conformations. The full configuration sum reads $\left(\beta=\left(k_{\mathrm{B}} T\right)^{-1}\right)$

$$
\int Q\left(x^{(N)}, y^{(N)}\right) \mathrm{d} x^{(N)} \mathrm{d} y^{(N)}=\left[\frac{1}{2 \pi} \int_{-\pi}^{+\pi} \mathrm{e}^{-\beta V(\phi)} \mathrm{d} \phi\right]^{N} .
$$

If the $\mathrm{C}$ atoms are restricted to the vertices of a lattice this expression simplifies even further:

$$
\int Q\left(x^{(N)}, y^{(N)}\right) \mathrm{d} x^{(N)} \mathrm{d} y^{(N)}=\left(\frac{1}{3}+\frac{2}{3} \mathrm{e}^{-\beta \epsilon}\right)^{N} .
$$

Abbreviating the right-hand side of (6) or (7) by $q^{N}$ we find from (5)

$$
Q\left(x^{(N)}, y^{(N)}\right)=q^{N} P\left(x^{(N)}, y^{(N)}\right)
$$

\section{The packing problem of hydrocarbon chains in a monolayer}

The results of the previous section will now be generalized to the case of $M$ hydrocarbon chains in a monolayer of area $\Omega=L^{2}$. The chains are supposed to have hard-core repulsion only, i.e. they cannot approach each other within a distance equal to $a$. Let $Q\left(r_{1}, r, \ldots, r_{M} ; N\right)$ denote the configuration sum of 
these chains with their head groups fixed at positions $r_{1}^{(0)}, r_{2}^{(0)}, \ldots, r_{M}^{(0)}$ and their endpoints at $\boldsymbol{r}_{1}, \boldsymbol{r}_{2}, \ldots, \boldsymbol{r}_{M}$. Our aim is to calculate $Q$ with the help of a method similar to the one employed in section 2 .

Projection of the $\mathrm{C}$ atoms on the $(x, y)$ plane gives rise to $M$ random walks in the plane. The steric constraints on the chain configurations are replaced by constraints on the random walks: for any fixed "time" $N$ no pair of random walks shall be closer than $a$. This "random walk" approximation implies that the chains do not fold back, which will be the case if the local number density of the head groups in the plane is not too low. This condition will be fulfilled if the overall density $\rho=M / \Omega$ is not too small, which is the case at the chain melting transition.

We are now in a position to calculate the configuration integral $Q\left(r_{1}, r_{2}, \ldots, r_{M} ; N\right)$. It can be shown in a straightforward way that $Q$ can be written in the form

$$
Q\left(r_{1}, r_{2}, \ldots, r_{M} ; N\right)=\frac{q^{N M}}{M !} F\left(r_{1}, r_{2}, \ldots, r_{M} ; N\right),
$$

where $F$ is the solution of the diffusion equation pertinent to this problem:

$$
\left[\frac{\partial}{\partial N}-D \sum_{i=1}^{M}\left(\frac{\partial^{2}}{\partial x_{1}^{2}}+\frac{\partial^{2}}{\partial y_{i}^{2}}\right)\right] F\left(r_{1}, r_{2}, \ldots, r_{M} ; N\right)=0
$$

under the initial condition

$$
\lim _{N \downarrow 0} F\left(r_{1}, r_{2}, \ldots, r_{M} ; N\right)=\prod_{l=1}^{M} \delta\left(r_{l}-r_{l}^{(0)}\right),
$$

and the boundary conditions

$$
F\left(r_{1}, r_{2}, \ldots, r_{M} ; N\right)=0, \quad \text { if }\left|r_{t}-r_{1}\right| \leqslant a \quad(i \neq j) .
$$

The solution can be written in terms of the orthonormal eigenfunctions $\boldsymbol{\phi}_{k}\left(\boldsymbol{r}_{1}, \boldsymbol{r}_{2}, \ldots, \boldsymbol{r}_{M}\right)$ of the Laplacian:

$$
\begin{aligned}
F\left(r_{1}, r_{2}, \ldots,\right. & \left.r_{M} ; N\right) \\
& =\sum_{k} \phi_{k}\left(r_{1}, r_{2}, \ldots, r_{M}\right) \phi_{k}^{*}\left(r_{1}^{(0)}, r_{2}^{(0)}, \ldots, r_{M}^{(0)}\right) \exp \left(-\lambda_{k} N\right),
\end{aligned}
$$

where $\lambda_{k}$ are the eigenvalues

$$
-D \sum_{i=1}^{M}\left(\frac{\partial^{2}}{\partial x_{l}^{2}}+\frac{\partial^{2}}{\partial y_{l}^{2}}\right) \phi_{k}\left(\boldsymbol{r}_{1}, \boldsymbol{r}_{2}, \ldots, \boldsymbol{r}_{M}\right)=\lambda_{k} \phi_{k}\left(\boldsymbol{r}_{1}, \boldsymbol{r}_{2}, \ldots, \boldsymbol{r}_{M}\right) .
$$

The boundary conditions are

$$
\phi_{k}\left(\boldsymbol{r}_{1}, \boldsymbol{r}_{2}, \ldots, \boldsymbol{r}_{M}\right)=0, \quad \text { if }\left|\boldsymbol{r}_{i}-\boldsymbol{r}_{\jmath}\right| \leqslant a \quad(i \neq j) .
$$

Combination of (9) and (13) gives

$$
\begin{aligned}
Q\left(r_{1}, r_{2}, \ldots, r_{M} ; N\right)= & \frac{q^{N M}}{M !} \sum_{k} \phi_{k}\left(r_{1}, r_{2}, \ldots, r_{M}\right) \\
& \times \phi *\left(r_{1}^{(0)}, r_{2}^{(0)}, \ldots, r_{M}^{(0)}\right) \exp \left(-\lambda_{k} N\right) .
\end{aligned}
$$


At this point it is convenient to impose periodic boundary conditions by identifying $\boldsymbol{r}_{1}$ with $\boldsymbol{r}_{1}^{(0)}$. The total configuration sum $Q_{M}(N)$ is then found by integrating (16) over all space coordinates:

$$
Q_{M}(N)=\frac{q^{N M}}{M !} \sum_{k} \exp \left(-\lambda_{k} N\right) .
$$

As follows from eq. (14), the quantities $\lambda_{k}$ in (17) correspond to the energy eigenvalues of a quantum gas of hard disks of diameter $a$ (with Boltzmann statistics), with $\hbar^{2} / 2 m$ replaced by $D$, and $\beta$ by $N$. The ground state energy $\lambda_{0}$ of this system has been calculated by Schick ${ }^{29}$ ) in the low density limit. Translated to our case it reads

$$
\frac{\lambda_{0}}{\Omega}=\frac{4 \pi D \rho^{2}}{\ln \left(\rho_{0} / \rho\right)} \quad\left(\rho_{0} / \rho \gg 1\right),
$$

with $\rho_{0}\left(\rho_{0}=2 /\left(a^{2} \sqrt{3}\right)\right)$ the close-packing density. We shall use (18) for all densities, but one should keep in mind that this extrapolation is only qualitatively correct.

At low densities the excitation spectrum is found to $b^{29}$ )

$$
\lambda_{k}=D k\left\{k^{2}+\frac{16 \pi \rho}{\ln \left(\rho_{0} / \rho\right)}\right\}^{1 / 2} \quad\left(\rho_{0} / \rho \gg 1\right) .
$$

This spectrum is free particle-like, apart from a sphere in $k$-space with a radius of the order $\left\{\rho / \ln \left(\rho_{0} / \rho\right)\right\}^{1 / 2}$. Hence, for low densities the spectrum may be taken to be identical to the free particle spectrum $\lambda_{k}^{(0)}\left(\lambda_{k}^{(0)}=D k^{2}\right)$. This leads to the approximate result

$$
\begin{aligned}
Q_{M}(N) & \cong \frac{q^{N M}}{M !} \exp \left(-\lambda_{0} N\right) \sum_{k} \exp \left(-\lambda \lambda_{k}^{(0)} N\right) \\
& =\frac{q^{N M}}{M !}\left(\frac{\Omega}{4 \pi N D}\right)^{M} \exp \left\{-\Omega \frac{4 \pi N D \rho^{2}}{\ln \left(\rho_{0} / \rho\right)}\right\} .
\end{aligned}
$$

The Helmholtz free energy per particle $f_{0}$ is given by

$$
f_{0}=-(\beta M)^{-1} \ln Q_{M}(N) \text {. }
$$

The pressure $p_{0}$ follows from

$$
p_{0}=\rho^{2}\left(\frac{\partial f}{\partial \rho}\right)_{\beta} .
$$

Combining eqs. (20)-(22) we find

$$
\beta p_{0}(\rho, \beta)=\rho+4 \pi N D\left[\frac{\rho^{2}}{\ln \left(\rho_{0} / \rho\right)}+\frac{\rho^{2}}{\ln ^{2}\left(\rho_{0} / \rho\right)}\right] .
$$

It seems appropriate to remark that the third term in (23) is of the same order of magnitude as the terms which we neglected in (18). This implies that this term may be dropped for low densities. 


\section{Inclusion of long-range attractive forces}

The monolayer is held together by long-range attractive forces. These forces have the character of van der Waals forces summed over the repeating units of the chains. In this section we shall assume that the range $\left(\gamma^{-1}\right)$ of the potential $V(r)$ of the attractive force is very large as compared to the diameter $(a)$ of a chain

$$
a \ll \gamma^{-1} \text {. }
$$

As a first step in the calculation of the effects of the attractive potential we divide the area $\Omega$ in cells, each of area $\delta^{2}$, such that ${ }^{30}$ )

$$
a \ll \delta \ll \gamma^{-1} \text {. }
$$

Let us label the cells by an index $i$ and denote the number of heads in cell $i$ by $M_{i}$. The $M_{t}$ satisfy the constraint $\Sigma_{i} M_{t}=M$. Because of the condition (25) the energy of a configuration $\left\{M_{1}\right\}$ equals

$$
\frac{1}{2} \sum_{i} V_{t, l} M_{i}\left(M_{i}-1\right)+\sum_{i<j} V_{i, j} M_{i} M_{l}=\frac{1}{2} \sum_{i, j} V_{l, j} M_{\imath} M_{l}-\frac{1}{2} \sum_{i} V_{t, l} M_{\imath}
$$

where $V_{l, j}$ denotes the interaction energy between a particle in cell $i$ and one in cell $j$. The canonical configuration sum equals

$$
\tilde{Q}_{M}=\sum_{\left\{M_{1}\right\}}^{\prime}\left(\prod_{l} Q_{M_{i}}\right) \exp \left(\frac{1}{2} \beta \sum_{l} V_{t, 1} M_{i}-\frac{1}{2} \beta \sum_{i, l} V_{i, l} M_{i} M_{l}\right)
$$

where $Q_{M_{i}}$ gives the configuration sum of $M_{l}$ chains without attractions, in a volume $\delta^{2}$. This quantity is given by (20) with $M$ replaced by $M_{1}$ and $\Omega$ by $\delta^{2}$. The prime indicates the constraint $\Sigma_{l} M_{t}=M$.

We shall approximate (27) by replacing the sum by its maximum term. Writing

$$
Q_{M}(N)=\exp \left(-\beta F_{0}\right),
$$

where $F_{0}$ is the Helmholtz free energy, the equation for the maximum term is found by differentiating the summand in (27) with respect to $M_{r}$. Setting this partial derivative equal to zero leads to

$$
\alpha+\frac{1}{2} \beta V_{0}-\beta \sum_{l} V_{\imath, j} M_{l}-\frac{\delta^{2}}{M_{\imath}}\left\{\beta p_{0}\left(\frac{M_{l}}{\delta^{2}}\right)+\beta f_{0}\left(\frac{M_{l}}{\delta^{2}}\right)\right\}=0
$$

where $V_{0} \equiv V_{l, l}$. The solution corresponding to a homogeneous density is found by putting

$$
M_{1}=M\left(\delta^{2} / \Omega\right) \text {. }
$$

This leads to the Helmholtz free energy density

$$
\begin{aligned}
\beta f(\rho, \beta) & =-\frac{1}{\Omega} \ln \tilde{Q}_{M} . \\
& =\beta \Omega^{-1} F_{0}(\rho, \beta)-\frac{1}{2} \beta V_{0} \rho-\frac{1}{2} \beta w_{0} \rho^{2},
\end{aligned}
$$


where

$$
w_{0} \equiv-\sum_{J} V_{i, j} \delta^{2}
$$

Using (23) and (31) the equation of state is found to be

$$
\begin{aligned}
\beta p(\rho, \beta)=\rho+4 \pi N D\left[\frac{\rho^{2}}{\ln \left(\rho_{0} / \rho\right)}+\frac{\rho^{2}}{\ln ^{2}\left(\rho_{0} / \rho\right)}\right] & -\frac{1}{2} \beta w_{0} \rho^{2}- \\
& \text { (+ Maxwell construction). }
\end{aligned}
$$

The method of ref. 30 automatically yields the Maxwell equal area construction; hence the present theory provides a description of the phase transition from first principles.

\section{Discussion}

The equation of state (33) predicts a critical point: it implies a critical temperature below which coexisting phases appear. Because the theory is essentially a mean-field theory, the critical exponents have classical values. Experiments [e.g. on dipalmitoyl phosphatidyl choline-lecithin ${ }^{31.32}$ )] do not show the full coexistence region, but only the onset of such a region. This makes it difficult to extract a critical point from these experiments. Several explanations have been proposed for the non-occurrence of a flat part in the experimentally determined isotherms: the effect of impurities ${ }^{5,8}$ ), the finite size of the trough in which the experiments are performed ${ }^{6}$ ), and the metastable nature of the liquid-condensed phase ${ }^{1}$ ).

Nagle $^{6}$ ) has estimated a critical point from the experiments of refs. 31 and 32. As our eq. (33) contains several adjustable parameters it is possible to fit the isotherm in the vicinity of Nagle's critical point. Taking for $\rho_{0}$ the value ${ }^{21}$ ) $\rho_{0}=0.5 \times 10^{15} \mathrm{~cm}^{-2}$ and fitting with the help of Nagle's $p_{\mathfrak{c}}$ and $\rho_{\mathrm{c}}$, the remaining parameters $\left(T_{\mathrm{c}}, D_{\mathrm{c}}\right.$ and $\left.w_{0}\right)$ are found to be of the correct order of magnitude. Quantitative agreement, however, is poor. This was to be expected for the following reasons: (i) expression (18) for the ground state energy of the hard disks Bose gas, which is correct at low densities, has been used at intermediate densities; and (ii) one cannot expect a mean-field type theory to lead to more than qualitative agreement with experiments. We are currently studying the possibility of improving on these approximations.

\section{Acknowledgements}

The authors would like to thank Dr. J. Hijmans and Prof. S.R. de Groot of the University of Amsterdam for critical reading of the manuscript. For one of us (A.J.K.) this work is part of the research programme of the "Stichting voor fundamenteel onderzoek der materie" (FOM), which is financially supported by 
the "Nederlandse organisatie voor zuiver-wetenschappelijk onderzoek" (Z.W.O.).

\section{References}

1) N L Gershfeld, Ann Rev Phys. Chem. 27 (1976) 349

2) R.H Tredgold, Advan Phys 26 (1977) 79

3) D. Chapman, Q. Rev. Biophys. 8 (1975) 185

4) D L Melchior and J.M Steim, Ann Rev Biophys Broeng 5 (1976) 205

5) S. Marcelja, Biochım. Biophys Acta 367 (1974) 165.

6) J F Nagle, J Membrane Biol. 27 (1976) 233

7) J F Nagle, J Chem. Phys 58 (1973) 252.

8) H.L. Scott, J Chem Phys. 62 (1975) 1347

9) M.J Janiak, D M Small and G G Shıpley, Biochemıstry 15 (1976) 4575.

10) G A Hawkıns and G.B. Benedek, Phys Rev Lett. 32 (1974) 524

11) M W Kim and D.S Cannell, Phys Rev A13 (1976) 411

12) S Ohki, J Theor Biol 15 (1967) 346

13) H.L Scott, J Theor Biol. 46 (1974) 241

14) D. Marsh, J Membrane Biol 18 (1974) 145.

15) R.E Jacobs, B Hudson and H C. Andersen, Proc Nat Acad Sc1 USA 72 (1975) 3993

16) J A McCammon and J.M Deutch, J Amer. Chem. Soc 97 (1975) 6675.

17) M.B Jackson, Biochemistry 15 (1976) 2555

18) B. Stoeckly, Phys Rev A15 (1977) 2558

19) J.F Nagle, Proc Roy Soc A337 (1974) 569.

20) J F Nagle, Phys Rev Lett 34 (1975) 1150

21) J F Nagle, J Chem Phys. 63 (1975) 1255

22) F W Wiegel, J Stat Phys 13 (1975) 515

23) F.W Wregel, Phys. Lett. 57A (1976) 393

24) F W Wiegel, Physica 89A (1977) 397

25) R G Priest, J Chem Phys 66 (1977) 722

26) F W Wiegel and A J Kox, Proc 13th IUPAP Conference on Statistical Physics, Haifa, 1977

27) P Flory, The Statıstıcal Mechanics of Chain Molecules (Interscience, New York, 1969)

28) S Chandrasekhar, Rev Mod Phys 15 (1943) 1

29) M Schick, Phys Rev A3 (1971) 1067

30) N G van Kampen, Phys Rev 135 (1964) A362

31) F Villalonga, Biochım. Bıophys Acta 163 (1968) 290

32) M C Phıllıps and D Chapman, Biochim. Biophys Acta 163 (1968) 301. 